\title{
Correction: Observational study assessing demographic, economic and clinical factors associated with access and utilization of health care services of patients with multiple sclerosis under treatment with interferon beta-1b (EXTAVIA)
}

Georgios Hadjigeorgiou, Efthimios Dardiotis, Georgios Tsivgoulis, Triantafyllos Doskas, Damianos Petrou, Nikolaos Makris, Nikolaos Vlaikidis, Thomas Thomaidis, Athanasios Kyritsis, Nikolaos Fakas, Xoulietta Treska, Clementine Karageorgiou, Stefania Sotirli, Christos Giannoulis, Dimitra Papadimitriou, loannis Mylonas, Evaggelos Kouremenos, George S. Vlachos, Dimitrios Georgiopoulos, Despoina Mademtzoglou, Michalis Vikelis, Elias Zintzaras

The eighteenth author's name is incorrectly spelled. The correct name is George S. Vlachos.

\section{Reference}

1. Hadjigeorgiou G, Dardiotis E, Tsivgoulis G, Doskas T, Petrou D, Makris N, et al. (2014) Observational Study Assessing Demographic, Economic and Clinical Factors Associated with Access and Utilization of Health Care Services of Patients with Multiple Sclerosis under Treatment with Interferon Beta-1b (EXTAVIA). PLoS ONE 9(11): e113933. doi:10.1371/journal.pone.0113933 PMID: 25419842

\section{OPEnACCESS}

Citation: Hadjigeorgiou G, Dardiotis E, Tsivgoulis G, Doskas T, Petrou D, Makris N, et al. (2015) Correction: Observational study assessing demographic, economic and clinical factors associated with access and utilization of health care services of patients with multiple sclerosis under treatment with interferon beta-1b (EXTAVIA). PLoS ONE 10(10): e0140308. doi:10.1371/journal. pone. 0140308

Published: October 6, 2015

Copyright: @ $\odot 2015$ Hadjigeorgiou et al. This is an open access article distributed under the terms of the Creative Commons Attribution License, which permits unrestricted use, distribution, and reproduction in any medium, provided the original author and source are credited. 